\title{
Improving Particle Filter Performance Using SSE Instructions
}

\author{
Peter Djeu and Michael Quinlan and Peter Stone
}

\begin{abstract}
Robotics researchers are often faced with realtime constraints, and for that reason algorithmic and implementation-level optimization can dramatically increase the overall performance of a robot. In this paper we illustrate how a substantial run-time gain can be achieved by taking advantage of the extended instruction sets found in modern processors, in particular the SSE1 and SSE2 instruction sets. We present an SSE version of Monte Carlo Localization that results in an impressive 9x speedup over an optimized scalar implementation. In the process, we discuss SSE implementations of atan, atan2 and exp that achieve up to a $4 x$ speedup in these mathematical operations alone.
\end{abstract}

\section{INTRODUCTION}

In robotics, it is often critical to process sensory data or to compute decisions in real-time, that is to say at the rate at which sensory data is captured. For example, a robot with a camera that captures frames at $30 \mathrm{~Hz}$ should complete its sense-act loop at the same rate or risk missing sensing opportunities. Sometimes this constrains the processing algorithms that can be used (e.g. Hough transforms may be ruled out entirely) and sometimes it constrains the quality of the processing (e.g. the number of particles that can be processed in a particle filtering algorithm). As a result, an important component of robotics is code streamlining and optimization. A common source of untapped potential for code optimization is the use of the vector unit on CPUs to perform efficient and parallel computation.

Since $1998^{1}$ most modern processors, including desktop and notebook/netbook processors, can perform SIMD (Single Instruction Multiple Data) operations which allow a single instruction stream to drive parallel computation. In particular both Intel and AMD support the SSE (Streaming SIMD Extension) instruction set and the follow-up instruction set SSE2 via an SSE vector unit [1]. Using the SSE vector unit allows the CPU to perform 4-wide operations in place of 1 wide operations; four additions can be performed in the time it usually takes to perform one. Under ideal settings, an SSE vector implementation can be up to $4 \mathrm{x}$ faster than the scalar implementation, and in cases such as the ones described in this paper, it can be even faster.

However, developing code that effectively uses the SSE instruction set has generally been restricted to graphics researchers or to the developers of heavily optimized libraries for specific tasks such as vision [2] and linear algebra [4]. While these libraries provide excellent performance on their intended tasks, the average roboticist has failed to take

All authors are in the Department of Computer Science, The University of Texas at Austin. djeu, mquinlan, pstone@cs.utexas.edu

${ }^{1}$ In 1998 AMD released the K6-2 processors, this was followed in 1999 by Intel's Pentium III processors advantage of the SSE vector unit in developing his or her code/algorithms.

In this paper we present ground-up SSE implementations of key functions required in the robotics domain (atan, atan2, exp) and apply them to a commonly used and practical robotics algorithm: Monte Carlo Localization (MCL) [3]. In our implementation we achieve an impressive 9x speedup, surpassing the ideal speedup of $4 \mathrm{x}$.

While this improvement in and of itself is noteworthy, one of the main objectives of this paper is to encourage and also instruct the robotics community on how to implement its own algorithms using the SSE instruction set. To help facilitate this task we are releasing the full source code for our MCL implementation, including the SSE components.

The remainder of this paper is structured as follows: Section II provides an overview of the basics of SSE. Section III introduces the Monte Carlo Localization algorithm and steps through the construction of the SSE version, with particular focus on the development of the SSE math extensions (such as atan2). Section IV will review and discuss the run-time performance results of both the overall MCL algorithm and the individual math operations. We will conclude and present future work in Section V.

\section{USING THE SSE INSRUCTION SET}

Traditional CPU instructions take single values as their operands. A typical floating point addition instruction has the following form, which we call 1 -wide addition.

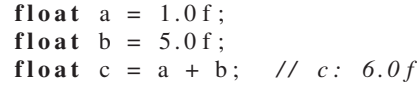

In SSE, the data types are expanded from 'float' to 'sse4Floats', which include the values of 4 separate floating points. When an addition is performed on two SSE operands, each of the 4 elements is independently summed. In other words, element 0 in the first operand is added to element 0 in the second operand, element 1 in the first operand is added to element 1 in the second operand, and so on. We will refer to this operation as a 4-wide addition.

In the following example, we will use a $\mathrm{C}++$ constructor for sse4Floats that takes 4 floating point values. The ' + operator is overloaded so that if both operands are sse4Floats, the SSE unit is invoked to perform a 4-wide addition.

sse4Floats a $=$ sse4Floats $(1.0 \mathrm{f}, 2.0 \mathrm{f}, 3.0 \mathrm{f}, 4.0 \mathrm{f})$;

sse 4 Floats $b=$ sse 4 Floats $(5.0 \mathrm{f}, 6.0 \mathrm{f}, 7.0 \mathrm{f}, 8.0 \mathrm{f})$;

sse4Floats $\mathrm{c}=\mathrm{a}+\mathrm{b} ; / / \mathrm{c}:(6.0 \mathrm{f}, 8.0 \mathrm{f}, 10.0 \mathrm{f}, 12.0 \mathrm{f})$

\footnotetext{
${ }^{2}$ The built-in SSE data type is actually '_-m128', however we encapsulate this type into a C++ class, which we call 'sse4Floats'.
} 
We will use the term 1 -wide and 4-wide to refer to mathematical operations that are performed analogously to the addition operations. The term scalar will be used interchangeably with 1-wide and vector interchangeably with 4-wide.

Apart from 4-wide floating point addition, the SSE vector unit also provides support for other arithmetic operations, such as subtraction, multiplication, division, minimum, maximum, and bitwise operations. The vector unit supports vectors of four 32-bit integers (aka ints) and has built-in support for addition, subtraction, and bitwise operations. Processors which support later versions of SSE also provide multiplication, minimum, and maximum operations for integers.

\section{A. SSE Masks}

One difficulty that occurs when using SSE is that a single instruction stream must be used for all processing. This is especially troubling when implementing algorithms that would normally require branching. Consider the following example, which returns the absolute value of $\mathrm{f}$.

float abs(float $\mathrm{f})\{$ return $(\mathrm{f}>=0.0 \mathrm{f})$ ? $\mathrm{f}:-\mathrm{f} ;\}$

When implementing a 4-wide version of absolute value for an sse4Floats consisting of the values $(-1.0 \mathrm{f},-2.0 \mathrm{f},+3.0 \mathrm{f}$, $+4.0 f)$, the first 2 elements need to be negated, while the last 2 elements do not. However, we must use the same instruction stream on all elements as per the definition of SIMD.

The solution is to evaluate both sides of the branch and to mask out the result from one branch and mask in the result from the other. The two results are then combined and only the masked-in result survives. We now revisit the scalar implementation to support this branching paradigm using bitwise operations. For brevity, the implementation of the two reinterpretation helper functions are not shown.

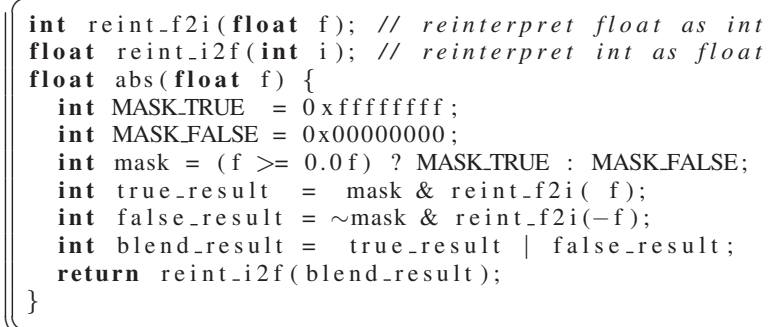

As you will notice, this version of absolute value still contains one branch when selecting the value of the variable 'mask', the value being either a 32-bit int with all bits set (the true mask) or a 32-bit int with all bits unset (the false mask).

The SSE vector unit solves this problem by mandating that all of its comparison operations produce either a true mask or a false mask in each element of the 4-wide result. In the following example, we generate an SSE mask by comparing the input value to another sse4Floats set completely to zero. We implement SSE masks using the sseMask data type, which is a $\mathrm{C}++$ class that encapsulates a built-in SSE data type representing a 4-wide mask.
sse4Floats a $=(1.0 \mathrm{f}, 0.0 \mathrm{f},-1.0 \mathrm{f},-2.0 \mathrm{f})$;

sse4Floats $b=(0.0 \mathrm{f}, 0.0 \mathrm{f}, 0.0 \mathrm{f}, 0.0 \mathrm{f})$;

sseMask mask $=(\mathrm{a}>=\mathrm{b})$;

// mask: (MASK_TRUE, MASK_TRUE, MASK_FALSE, MASK_FALSE)

This sseMask can then be used in a 4-wide blend operation which takes three operands: an sseMask, a true operand, and a false operand. The return value is an sse4Floats that is set to the value of the true operand at all elements where the sseMask had value MASK_TRUE. The return value is set to the false operand at all other locations. The operators '\&', '|', and ' $\sim$ ' are overloaded to invoke the corresponding bitwise operations in the SSE unit.

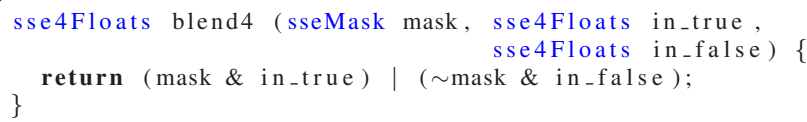

The following is an SSE implementation of absolute value. The ' $>=$ ' operator is overloaded to invoke the greater-thanor-equal-to comparison operator in the SSE unit, while the '-' operator is overloaded to invoke the unary negation operator in the SSE unit.

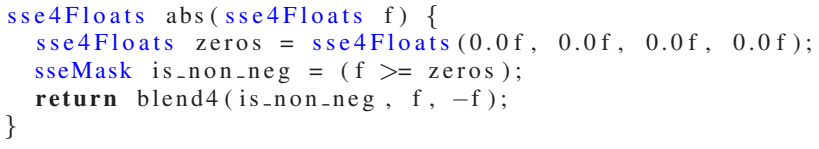

To summarize, masking allows us to conditionally perform operations on only certain elements within an sse4Floats rather than on all elements. Masking comes with a cost, however, since both the true input and the false input must be evaluated whenever a mask is used. In scalar code, work can often be saved by descending down only one branch of a conditional, while in pure SSE code both sides of the branch must always be taken, even in cases where the sseMask consists of 4 MASK_TRUE's or 4 MASK_FALSE's.

\section{B. Reduction Operations}

It is often necessary to perform an operation which reduces the 4 values within an sse4Floats to a single floating point value.

$$
\begin{array}{ll}
\text { sse } 4 \mathrm{Floats} & \mathrm{a}=\operatorname{sse} 4 \mathrm{Floats}(1.0 \mathrm{f}, 2.0 \mathrm{f}, 3.0 \mathrm{f}, 4.0 \mathrm{f}) \\
\text { float } & \mathrm{b}=\text { reduce_add }(\mathrm{a}) ; / / b: 10.0 \mathrm{f}
\end{array}
$$

These operations which work internally within a single sse4Floats are known as reduction operations. Reduction operations are typically slower than their 4-wide counterparts; however they are usually needed only sparingly. For example, the following block of code returns the average value of all floats in an array of sse4Floats. Notice that only one reduction operation is needed.

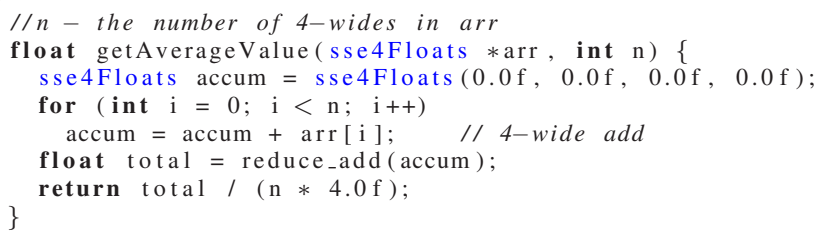




\section{SSE Performance}

Using the SSE vector unit to perform 4-wide operations in place of 1-wide operations allows us to perform four arithmetic operations in the time it would take to perform one. Under ideal settings, an SSE implementation can be up to $4 \mathrm{x}$ faster than the scalar implementation. We will refer to the ratio of the scalar version's run-time to the SSE version's run-time as parallel speedup.

There are many reasons why parallel speedup falls below 4x. Often, reformatting data to conform to an SSEcompatible layout is expensive, as is converting from SSE format to an output format. More generally, we must operate within the constraints of Amdahl's Law, where any part of an algorithm that cannot be parallelized will limit the overall speedup. In applications where data is not readily available in multiples of 4, some elements in the SSE vector will be left empty, which further limits speedup. Finally, for algorithms that have deep conditional branches, where one or both sides of the branch contain a significant amount of work, the SSE version will suffer because it must perform the work on both sides of the branch, compared to the scalar version which only needs to execute the taken branch.

It is possible, however, to achieve a parallel speedup that is greater than the ideal speedup of $4 \mathrm{x}$, which is evidenced in this work. The reason this is possible is because of a fundamental difference in the hardware that evaluates SSE code and scalar code which exists on many platforms. For example, on many Intel chips the ALU that performs SSE floating point operations is faster than the ALU which handles scalar floats since the latter is based on the aging design of the x87 math co-processor. In other words, a parallel speedup that exceeds $4 \mathrm{x}$ is possible due to a combination of good data parallelism (SIMD) and intrinsic speedup due to using better hardware (the SSE ALU is faster than the scalar ALU).

In our experience, the best time to switch from a scalar implementation to an SSE implementation is when the scalar algorithm requires performing arithmetic operations independently but identically over a large data set. For example, an algorithm that evaluates the $\exp (\mathrm{x})$ function on every element of an array is a prime candidate for conversion to SSE.

\section{SSE AND MONTE CARLO LOCALIZATION}

\section{A. Monte Carlo Localization (Particle Filters)}

For the purpose of this paper we will describe the implementation of a simple version of Monte Carlo Localization (MCL) [3]. We hope that our description provides others with the necessary framework to begin including SSE in deployable real-world implementations. We have chosen to keep the example as straightforward as possible (see Algorithm 1) in an attempt to clearly demonstrate the modifications needed to use the SSE instruction set. Notice that in Line 10 of our implementation, we compute particle likelihoods by accumulating the exponent of the probability in the inner loop and perform the exponentiation later in the estimatePose() helper function (Line 13). Both versions of the MCL algorithm (scalar and SSE) benefit from this optimization.

Our MCL implementation will be taking observations (measurements) in the form of the distance $(\mathrm{mm})$ and bearing (radians) to known reference objects. Figure 1 presents the output of the algorithm when either one or three objects are being observed.
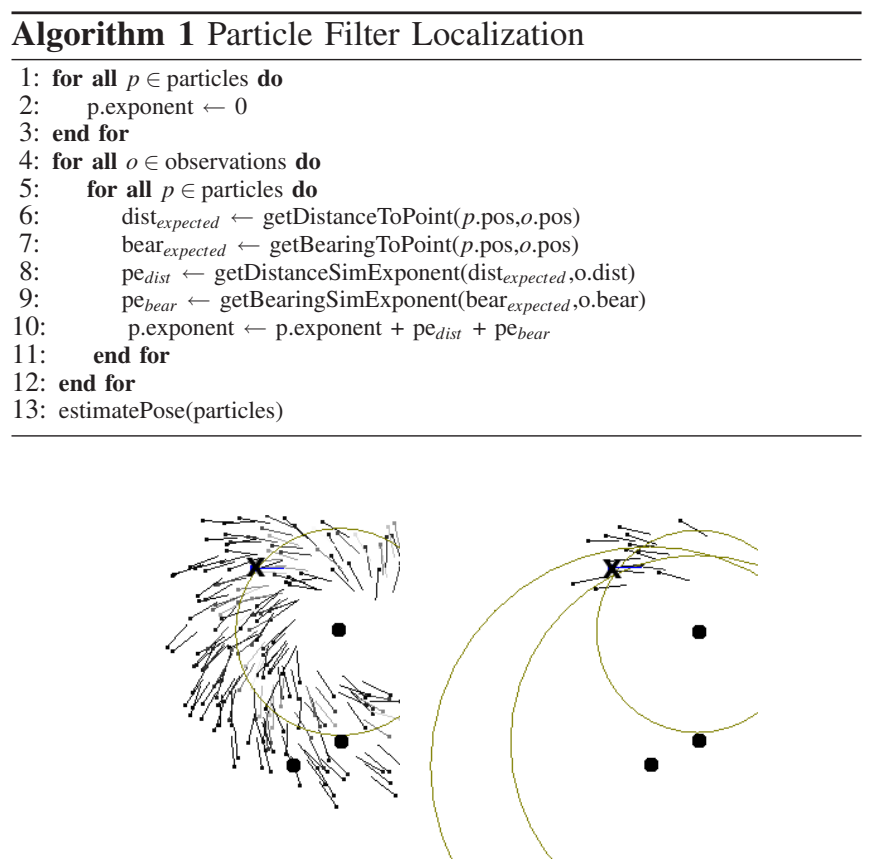

Fig. 1. Example Particles: In the left image, the robot (drawn as an X) observes a single object. One reference object is not enough for localization, which results in the most likely particles forming a ring around that object. In the image on the right, the robot is observing all three objects, resulting in the most likely particles clustering around the robot's true location.

\section{B. SSE Math Extensions}

The processor's built-in support for SSE, which in this paper is considered to be the SSE instruction sets 'SSE1' and 'SSE2', is sufficient for implementing many algorithms [1]. We choose to limit our scope to these two instruction sets in order to support older processors. For Monte Carlo localization, we need to evaluate $\operatorname{atan} 2(y, x)$ and $\exp (x)$ for 4-wide inputs and return results that are also 4-wide. Neither function is part of the built-in instruction set so we will provide details on our implementations.

\section{SSE Data Structures}

For our SSE implementation, we use SSE data structures to represent four particles at a time. Our 4-wide particle data structure represents 4 separate particles, where each particle consists of an $\mathrm{x}$ coordinate, a $\mathrm{y}$ coordinate, an orientation (aka bearing), a probability exponent, and a probability.

\begin{tabular}{|ll|} 
class Particle_4Wide & \\
sse4Floats $x ;$ & $/ /$ 4-wide $x$ coordinate \\
sse4Floats $y ;$ & $/ /$ 4-wide y coordinate \\
sse4Floats o; & $/ /$ 4-wide bearing \\
sse4Floats exponent $;$ & $/ /$ prob exponent \\
sse4Floats prob; & $/ /$ equal to exp (exponent) \\
\hline
\end{tabular}


We reuse the basic outline for a particle filter from Algorithm 1 for our SSE implementation, where we overload our functions from our scalar version to recognize and return an sse4Floats in place of a float and a Particle_4Wide in place of a Particle.

Another modification we need for the SSE particle filter is to expand the observations. Normally, observations and particles are both 1-wide. However, now that particles are 4-wide, the observation must also be 4-wide. This is accomplished by duplicating the data from one observation across all elements of a 4 -wide in a process called expansion. The expanded observation can now be compared against the 4-wide particle. To implement expansion, we will use the function, expand(float $\mathrm{x}$ ), which creates an sse4Floats from a single float.

\section{Bearing To A Point}

Calculating the expected bearing from a single particle to an object (Line 4 in Algorithm 1) requires the following code. The normalizeAngle() function converts an angle from the interval $(-\infty, \infty)$ to the interval $[-\pi, \pi]$.

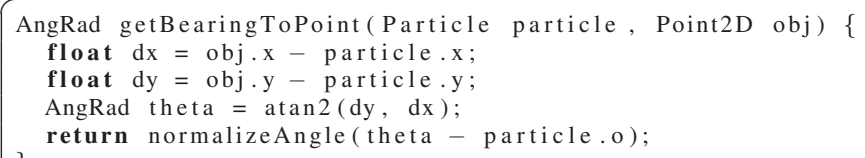

Notice that we need to perform an atan2 during the bearing computation for every combination of observation and particle. To implement $\operatorname{atan} 2(y, x)$, we will first implement the helper function $\operatorname{atan}(\mathrm{x})$. In the following sections, we describe our SSE implementations for both of these functions.

1) Function 1: atan: The $\operatorname{atan}(\mathrm{x})$ function determines the angle theta on $[-\pi / 2, \pi / 2]$ for which $\tan ($ theta $)=x$. Our implementation computes atan by evaluating the first seven terms in Euler's power series for atan. This series expansion converges more rapidly than the more commonly used power series for atan. The helper method__atan_rd(x) evaluates the actual power series on the reduced domain $(\mathrm{rd})$ of $[0,1]$, which guarantees that the power series will converge. In order to convert a generic input to _-atan_rd(x)'s reduced domain, we use the following two trigonometric identities:

Trig Ident 1: $\operatorname{atan}(\mathrm{x})=-\operatorname{atan}(-\mathrm{x})$

Trig Ident 2: $\operatorname{atan}(x)=P I / 2-\operatorname{atan}(1 / x)$

These two identities can be used to transform any input $\mathrm{x}$ from the real number line to the interval $[0,1]$. We will call this process of compressing inputs to a smaller interval domain reduction. When domain reduction is used, it usually has a corresponding range expansion. In this case, we first apply domain reduction in atan() in preparation for the call to _-atan_rd(x). After__atan_rd(x) is evaluated, we use range expansion to fix the sign of the return value from __atan_rd(x) if we used the first trigonometric identity during domain reduction. The second part of range expansion is to subtract from $\pi / 2$ if we used the second trigonometric identity during domain reduction. We provide a further optimization by subtracting from either $-\pi / 2$ or $\pi / 2$ to guarantee that the output from $\operatorname{atan}(\mathrm{x})$ is in the range $[-\pi / 2, \pi / 2]$.

In order to improve the accuracy of the __atan_rd(x) function, we provide a small fix for values of $\mathrm{x}$ that are near 0 . These values will undergo floating point underflow during self-multiplication, which drives the output of __atan_rd(x) to zero. To alleviate this problem, we simply return the value of $\mathrm{x}$ for all values for which the reference implementation of $\operatorname{atan}()$ from math.h also returns the value $\mathrm{x}$. The largest such value of $\mathrm{x}$ is about 0.000352 .

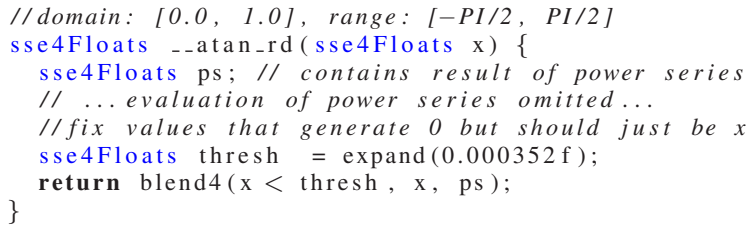

2) Function 2: atan2: The $\operatorname{atan} 2(\mathrm{y}, \mathrm{x})$ function is a more robust version of $\operatorname{atan}(\mathrm{x})$. The $\operatorname{atan} 2(\mathrm{y}, \mathrm{x})$ function returns the angle theta on $[-\pi, \pi]$ that is formed by the positive $\mathrm{x}$ axis and the line segment connecting the origin to the point $(x, y)$. The $\operatorname{atan} 2(\mathrm{y}, \mathrm{x})$ function is used during bearing computations in the particle filter.

Our implementation uses $\operatorname{atan}(\mathrm{x})$ as a helper method by computing $y / x$ and then invoking $\operatorname{atan}(\mathrm{x})$. The raw result from $\operatorname{atan}(\mathrm{x})$ is then put through a range expansion based on the quadrant in which the input to $\operatorname{atan} 2(y, x)$ lies. The range expansion takes the return value of $\operatorname{atan}(\mathrm{x})$ and converts it from either quadrant 4 to quadrant 2 by adding $\pi$ or from quadrant 1 to quadrant 3 by subtracting $\pi$, as necessary.

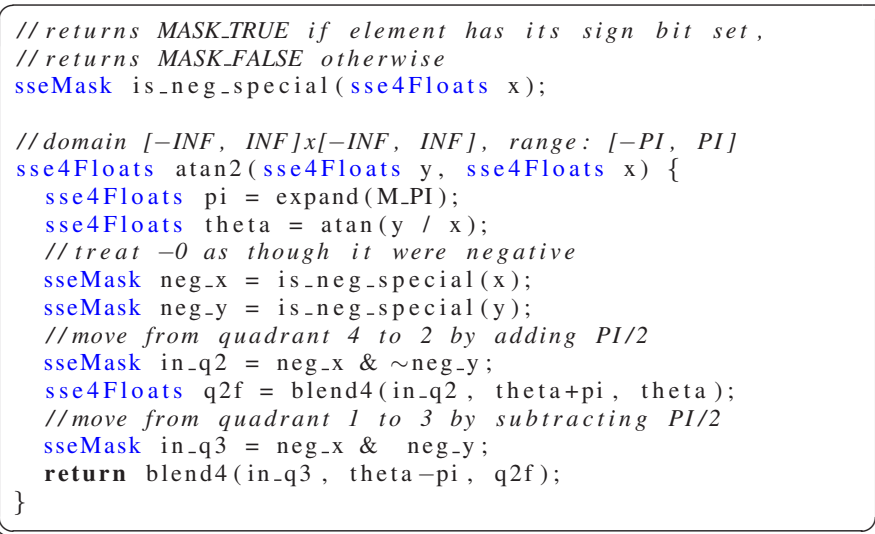




\section{E. Distance/Bearing Similarity}

Typically, the distance and bearing similarity are computed by evaluating an exponential $e^{x}$ for each similarity within the inner loop of the particle filter. These similarities are then accumulated into a total likelihood for the particle via multiplication. However, we can reduce the number of exponentiations that are needed by deferring the exponentiation until pose estimation for the robot. To do so, we simply sum the exponents of the similarity values within the inner loop of the particle filter (Lines 8-10 in Algorithm 1). Later, we produce a mathematically identical result by evaluating the exponential in the call to the estimatePose() helper function (Line 13 of Algorithm 1). Note the use of $\exp (\mathrm{x})$ in Line 4 of Algorithm 2.

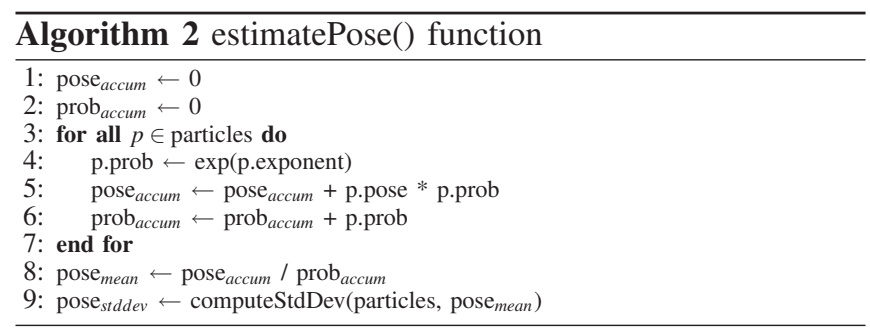

By deferring the evaluation of $\exp (\mathrm{x})$ until estimatePose(), we can reduce the number of exponentiations needed by a factor of $\mathrm{N}$, where $\mathrm{N}$ is the number of observations from Algorithm 1. In the remainder of this section we describe our SSE implementation of $\exp (\mathrm{x})$.

1) Function 3: exp: The $\exp (\mathrm{x})$ function evaluates $e^{x}$. The $\exp (\mathrm{x})$ function is used for computing likelihoods in the particle filter. Our implementation for $\exp (\mathrm{x})$ is broken down into three helper methods, _-_exp_rd(x) and its helper methods __exp_exponent(ipart) and __exp_mantissa(x).

The first step in the $\exp (\mathrm{x})$ function is to pass its input to __exp_rd(x), which works on the reduced domain of approximately $(-87.3,88.7)$. Inputs outside of this domain are trivial to evaluate and will be handled by the main $\exp (\mathrm{x})$ function later.

The _-exp_rd(x) function evaluates $e^{x}$ by evaluating the result in two parts based on the composition of a 32-bit float. Recall that a 32-bit float consists of 1 bit for the sign, 8 bits for the exponent, and 23 bits for the mantissa. The first part of our algorithm determines the correct 8 bits for the exponent in the helper function __exp_exponent(ipart). The second part of our algorithm determines the correct 23 bits for the mantissa in the helper function __exp_mantissa(x). These results are combined and returned by the function __exp_rd(x). Finally, for any input that exceeds the reduced domain of __exp_rd(x), the main $\exp (\mathrm{x})$ function masks in either 0.0 or $\infty$ based on whether the input is less than or greater than the reduced domain, respectively.

It should be noted that _-_exp_mantissa(x) evaluates $e^{x}$ on the reduced domain $\left[0.0, \log _{2}(e)\right]$ based on a special seventerm power series that has been fitted for high accuracy on the reduced domain. The coefficients differ from the ones used in the standard series expansion for $e^{x}$ so we refer the reader to our code release.

The following code uses the new data type sse4Ints. This data type is a C++ class that encapsulates an '-_m128i', which represents four 32-bit integers rather than four 32-bit floats. For this class we have overloaded the $<<$ operator to invoke the SSE unit's bitwise left shift instruction.

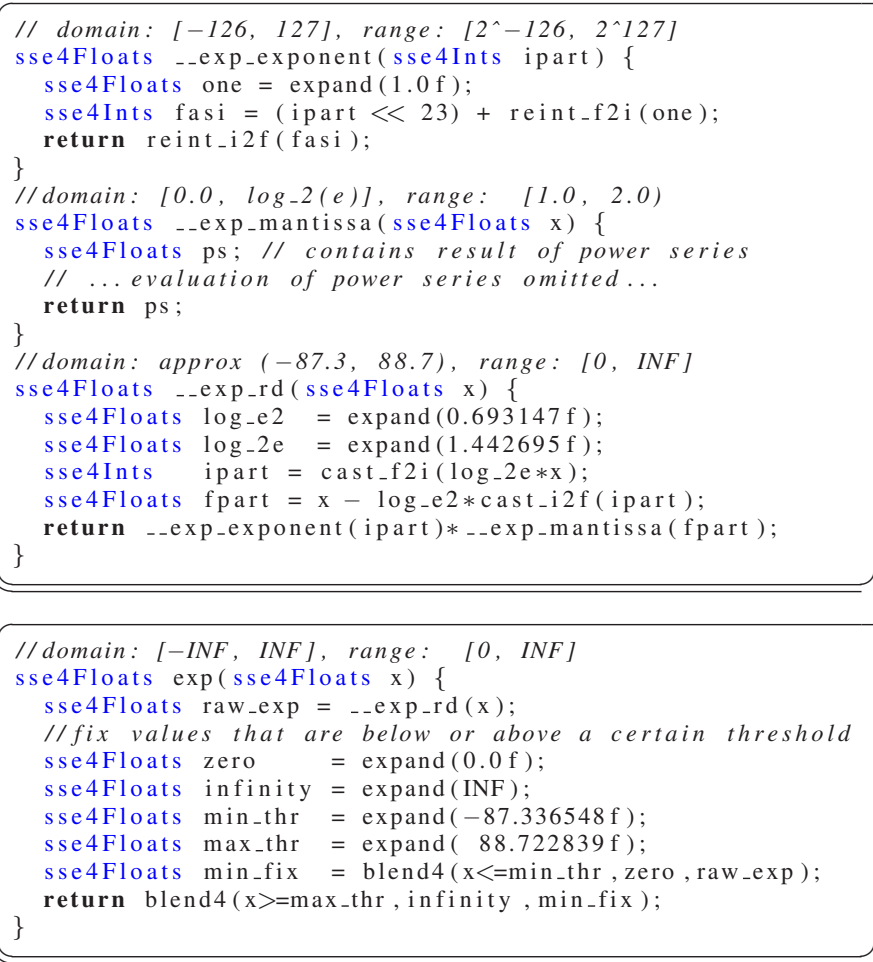

\section{RESULTS}

Three experiments were performed to test various aspects of run-time performance. The first experiment tests how well both the scalar and SSE particle filters scale with the number of particles as well as their respective accuracy. The second experiment examines how many particles each version can support when there is a hard real-time constraint. The third experiment isolates the performance of the SSE versions of the advanced math operations. All experiments were conducted on a $2.66 \mathrm{GHz}$ Core 2 Duo using the Intel Compiler version 10.1.029.

\section{A. Scaling Experiment}

The purpose of this experiment is to measure the runtime performance the SSE and scalar particle filters while varying the number of particles. Maximum optimization settings were used for both the scalar and SSE versions. These experiments were run with noise-free observations and with three observations at each measurement update. The results are shown in Table I and Figure 2.

We achieve a consistent parallel speedup of at least 7.6x in all of our runs, surpassing the ideal limit of $4 \mathrm{x}$ due to a combination of good data parallelization and use of better ALUs in the SSE version. This result provides strong motivation for using an SSE implementation over a scalar implementation. 
As expected, localization error decreases as the number of particles increased. We reach a saturation point at $\geq 16384$ particles, where additional particles no longer improve the accuracy of the pose estimate. Notice that for sufficiently low $n$, the particles are too sparsely distributed and all have 0 likelihood. When this occurs, the pose of the robot cannot be estimated and the error is infinite, which is represented by $N / A$ in the table.

\begin{tabular}{||c|c|c|c|c|c||}
\hline$n$ & Scalar & SSE & Speed-up & \multicolumn{2}{|c||}{ Error } \\
& $(\mathrm{s})$ & $(\mathrm{s})$ & & $\mathrm{mm}$ & $\mathrm{rad}$ \\
\hline 4 & 0.000023 & 0.000003 & 7.7 & $N / A$ & $N / A$ \\
16 & 0.000028 & 0.000003 & 9.3 & $N / A$ & $N / A$ \\
64 & 0.000047 & 0.000006 & 7.8 & 1590.0 & 0.513 \\
256 & 0.000116 & 0.000015 & 7.7 & 820.0 & 0.307 \\
1024 & 0.000403 & 0.000053 & 7.6 & 359.6 & 0.155 \\
4096 & 0.001607 & 0.00020 & 7.7 & 171.0 & 0.0742 \\
16384 & 0.006647 & 0.000831 & 8.0 & 109.0 & 0.0447 \\
65536 & 0.031178 & 0.003308 & 9.4 & 108.0 & 0.0446 \\
262144 & 0.125763 & 0.013664 & 9.2 & 110.0 & 0.0450 \\
1048576 & 0.460547 & 0.057089 & 8.1 & 109.0 & 0.0446 \\
\hline
\end{tabular}

TABLE I

SCALING RESULTS OVER $n$ PARTICLES. ERROR IS THE AVERAGE ERROR OVER 100 RANDOM CONFIGURATIONS OF PARTICLES.

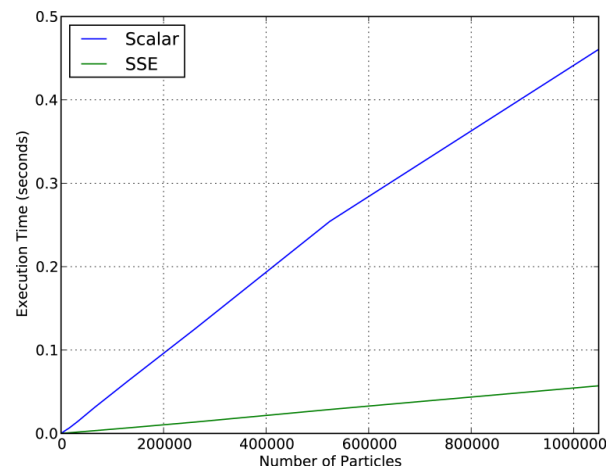

Fig. 2. Timing results for our scalar and SSE implementations of MCL.

\section{B. Real-time Experiment}

This experiment determines the number of observations we can process at real-time rates without ever going over a hard deadline of $30 \mathrm{~Hz}$. We use 16384 particles, the saturation point determined in the scaling experiment. We vary the number of observations until we find the largest value that can meet the real-time deadline for 500 consecutive invocations of the particle filter, where each invocation is given a new set of observations.

The scalar version of the particle filter can handle up to 20 observations in this test, while the SSE version can handle 157 observations. The observed increase in particles by using SSE is a factor of 7.9x, which closely matches the speedup of 8.0x for 16384 particles from Table I.

\section{Performance of SSE Math Functions}

In this section we present the raw performance of the SSE math algorithms (atan, atan2 and exp). Table II summarizes the performance. Notice the $\exp ()$ function approaches the optimal 4x speedup.

\begin{tabular}{||c|c|c|c||}
\hline Function & Scalar $(\mathrm{sec})$ & SSE $(\mathrm{sec})$ & Speedup \\
\hline atan & 38.086196 & 20.303419 & 1.8769 \\
atan2 & 65.351570 & 28.582232 & 2.2644 \\
$\exp$ & 19.501226 & 4.967288 & 3.9259 \\
\hline
\end{tabular}

TABLE II

SSE Math Results: $\exp$ TESTS ALL FlOATS ON THE INTERVAL [-80.0, 80.0]. atan TESTS ALL FLOATS ON THE INTERVAL $[-\infty, \infty]$. atan 2 TESTS ALL PAIRS OF FLOATS ON THE UNIT CIRCLE.

\section{CONCLUSION AND FUTURE WORK}

This article described the construction of an SSE version of a Monte Carlo Localization algorithm, including developing SSE mathematical operations that are frequently required in the robotics domain. We have shown that a 9x speedup is possible over optimized scalar code. This speedup allows us to either a) process the same number of particles in less time or b) increase the accuracy of our localization by using a greater number of particles. The ability to support more particles also reduces the need for MCL algorithms to perform sophisticated and often expensive particle resampling.

Almost all processors on the market directly support SSE. Our results demonstrate that there is significant untapped potential in the SSE vector unit on these processors. To facilitate the exploration of this potential by the robotics community, we have developed and will open-source the implementation of our SSE particle filter and underlying components.

In future work we will extend our library to additional mathematical operations and sub-routines. We hope to roll out fully vectorized versions of common robotics algorithms and to take advantage of SSE in loop-heavy tasks such as processing lidar sensor readings.

We refer the reader to our source code release for additional implementation details not covered in this paper:

http://www.cs.utexas.edu/users/mquinlan/mcl_sse.html

\section{ACKNOWLEDGEMENTS}

We would like to thank Warren Hunt for contributing the design and implementation of the exp function. This research is supported in part by grants and funding from the National Science Foundation (CNS-0615104), DARPA (FA8750-05-2-0283 and FA865008-C-7812), the Federal Highway Administration (DTFH61-07-H00030), General Motors. and Intel Corporation. The authors also thank Donald Fussell and Katherine Bontrager for comments and feedback on the paper.

\section{REFERENCES}

[1] Intel Corporation, Getting started with SSE/SSE2 for the Intel Pentium 4 Processor, White paper, http://www.developers.net/intelisnshowcase/view/116.

[2] Intel Open Source Computer Vision Library (OpenCV), http://opencvlibrary.sourceforge.net.

[3] Sebastian Thrun and Wolfram Burgard and Dieter Fox, Probabilistic Robotics (Intelligent Robotics and Autonomous Agents, The MIT Press, 2005.

[4] R. Clint Whaley and Jack Dongarra, Automatically Tuned Linear Algebra Software, Ninth SIAM Conference on Parallel Processing for Scientific Computing, 1999, Latest library at http://mathatlas.sourceforge.net/. 